\title{
Green Algae Diagnosis and Management in Low Land Paddy Fields of Cauvery Delta Zone, Tamil Nadu, India
}

\author{
M. Jeya Bharathi ${ }^{1^{*}}$, K. Rajappan ${ }^{2}$ and M. Raju ${ }^{3}$ \\ Tamil Nadu Rice Research Institute, Aduthurai, India \\ *Corresponding author
}

\section{Keywords}

Green algae, Laboratory and field experiments, $\mathrm{CuSO}_{4}$

Article Info

Accepted:

26 January 2021

Available Online:

10 February 2021

\begin{abstract}
A B S T R A C T
Rice is a prime food crop for Asian countries. Wet land rice cultivation contributes maximum grain yield than dry land rice. Cauvery delta is a predominant area for rice cultivation in Tamil Nadu. Green algae growth during Kuruvai (June -August) season is a serious problem in wet land rice. Laboratory and field experiments were conducted to find out the remedial measures. The results of soil and water analyses showed that use of bore well water and dumping of phosphatic fertilizers leads to salt accumulation which favours the algal growth. The results of the laboratory experiment revealed that the $\mathrm{CuSO}_{4}$, londox power, propiconazole and hexaconazole showed moderate inhibition on $5^{\text {th }}$ day after treatment. The findings from field experiment indicated that use of conoweeder and $\mathrm{CuSO}_{4}$ drenching at the rate of $2.5 \mathrm{~kg} / \mathrm{ha}$ when green algae appearance has just noticed or $5.0 \mathrm{~kg} / \mathrm{ha}$ when severe growth occurred is effective in managing the green algae. Biofertilizers application, crop rotation, green manure trampling to be practised to control the algae growth not rectified by $\mathrm{CuSO}_{4}$ application.
\end{abstract}

\section{Introduction}

Rice (Oryza sativa L.) is a staple food for more than half of the world's population. Globally, rice is grown on 161 million hectares, with an average annual production of 678.7 million tonnes (Bargali et al., 2009; Vibhuti et al., 2015). India is the largest rice growing country, accounting for about onethird of the world acreage under the crop (Bargali et al., 2007 \& 2009; Rajkumar, 2013). It is one of the most widely grown crops in Tamil Nadu state of India under million hectares.

Green algae or slime is a perpetual problem in wet land rice cultivation. It is prevalent in the water as a large slab of algae, that covers the surface and smothers the emerging rice crop. It thrives well where there is plenty of nutrients in the water and plentiful sunlight encourages photosynthesis (Coptrol, Rural chemicals industries (Australia) private limited, 1957). Green algae (singular: green alga) are a large, informal, grouping of algae consisting of the chlorophyte and charophyte/Streptophyta, which are now placed in separate divisions, as well as the more basal Mesostigmatophyceae and Chlorokybophyceae (Sánchez-Baracaldo et al., 2017). Embryophytes or green algae have 
emerged from the charophytes (Jeffrey et al., 2004). Green algae have chloroplasts that contain chlorophyll $a$ and $b$ giving them a bright colour as well as the accessory pigments beta carotene and xanthophylls in stacked thylakoids (Hoek et al., 1995).

The cell walls of green algae usually contain cellulose and they store carbohydrate in the form of starch (Judd et al., 2002). Like land plants, green algae undergo open mitosis without centrioles (Raveen et al., 2005).

Algae are commonly present in irrigation water and generally the rate they multiply is balanced by natural degeneration. However, when the balance is upset through increased sunshine or by the introduction of extra nutrients, the rate of growth increases and the algae blooms become a problem in rice growing.

Before algae are visually detected a lot of damage has already been done to the crop at its most vulnerable stage of growth (Coptrol, Rural chemicals industries (Australia) private limited, 1957). With this background the current investigation was focused to find out the reason behind the algae growth and to device management practices to control algae growth in wet land rice fields.

\section{Materials and Methods}

\section{Laboratory experiment}

Laboratory experiments were carried out at Tamil Nadu Rice Research Institute, Aduthurai. Green algae were collected from infected rice fields. Ten gram of algae was placed in the Petri dish. Fungicides, weedicides and algicides were added at the rate of $2 \mathrm{ml} /$ plate each separately, closed with lid and left for 7 days. The observations were recorded every day after incubation.

\section{Preliminary field experiment}

A field experiment was conducted at Tamil Nadu Rice Research Institute, Aduthurai during June to August 2015 (Kuruvai season) to determine green algae management practices under wet land rice ecosystem. Experiments were laid out in a randomized block design with 11 treatments and 3 replications. Fertilizers were applied at the rate of 150: 50: $50 \mathrm{~kg} \mathrm{NPK/ha.} \mathrm{N} \mathrm{was} \mathrm{applied}$ $50 \%$ as basal and two top dressings of $25 \%$ each at the time of active tillering and panicle initiation stages. The size of the experimental plot adopted with $3 \times 4 \mathrm{~m}$ and 2 seedlings / hill. Soil sample $(50 \mathrm{~g})$ was drawn at 30,60 and 90 days after planting (DAP) for enumeration of bacteria (Allen, 1953), fungi (Martin, 1950) and actinomycetes (Allen, 1953). The survival of Azospirillum (Okon et al., 1977) and Phosphobacteria (Sperber, 1958) in the rhizosphere of ADT 43 was estimated by MPN and standard plate count method (Parkinson, 1971) respectively. The Pseudomonas population was estimated using King's B (Kings et al., 1954) medium. Available N, P and $\mathrm{K}$ were estimated by alkaline permanganate method (Subbian and Asija, 1956), Olsen's method (Olson et al., 1954) and flame photometer method (Standford and English, 1949) respectively. The grain yield was recorded at the time of harvest (Fig. 1 and 2).

a Total bacteria were enumerated by serial dilution plating method on soil extract agar medium (James, 1958).

b Total culturable fungi were enumerated by serial dilution plating method as described by Parkinson et al., (1971)

c Total diazotrophs were enumerated by the procedure as described by Rennie (1981). 


\section{Confirmatory field experiment}

Field experiments were conducted during Kuruvai season in two places viz., Aduthurai and Maharajapuram village located at Thanjavur district during June to August 2016. Fertilizers were applied as in the case of preliminary field experiment. The experiments were laid out in a randomized block design with 12 treatments and 3 replications. The plot size was $4 \mathrm{~m} \times 3 \mathrm{~m}$ with $20 \times 15 \mathrm{~cm}$ spacing having 2 seedlings / hill. ADT 45 variety was used in this experiment

\section{Soil and water physical parameter analysis}

Soil and water sample were collected from algae noticed fields for analysing the reason behind the over algae growth. Available N, P and $\mathrm{K}$ were estimated as described earlier. Micronutrients viz. $\mathrm{HCO}_{3}, \mathrm{~S}, \mathrm{Cl}, \mathrm{Ca}, \mathrm{Mg}, \mathrm{Na}$ and $\mathrm{K}$ were estimated using Atomic Adsorption Spectrophotometer (AAS) method and expressed in ppm. The electrical conductivity (EC) and $\mathrm{pH}$ were estimated using $\mathrm{EC}$ and $\mathrm{pH}$ meter respectively.

\section{Results and Discussion}

Effect of fungicides, weedicides and algicides on algae growth under laboratory and field conditions

The laboratory experiments revealed that the application of propiconazole, hexaconazole, $\mathrm{CuSO}_{4}$ and londox power showed moderate inhibition on algae growth at $5^{\text {th }}$ day of incubation. The algae became shrunken and black in colour. Other chemicals don't have negative impact on the algae growth as given below (Table 1). Field experiment results revealed that $\mathrm{CuSO}_{4}$ application inhibited algal growth $100 \%$, whereas, londox power exhibited $50 \%$ inhibition of algal growth (Table 2) while, other chemicals don't have inhibition on algal growth. Chemicals réaction on algae under in vitro and in vivo conditions were significantly different.
Eventhough $\mathrm{CuSO}_{4}$ inhibit algae growth under field conditions, it was not observed in labortory conditions. This might bé due to the change in the soil $\mathrm{pH}$ due to $\mathrm{CuSO}_{4}$ application.

\section{Effect of fungicide, weedicide and algicide application on nutrient availability and microbial population under field conditions}

Bispyriphos sodium and butachlor application increased soil available $\mathrm{N}$ content at the rate of 831.25 and $650 \mathrm{~kg} / \mathrm{ha}$ respectively. On the other hand, butachlor and $\mathrm{CuSO}_{4}+$ CaOapplication increased soil available $\mathrm{P}$ (142 kg/ha). Bispyriphos sodium and propiconazole application increased soil available $\mathrm{K}(775 \mathrm{~kg} / \mathrm{ha})$ at $90 \mathrm{DAT}$ as given in the Table 3. This might be due to the changes in soil acidic and alkali status by the application of the chemicals. There is no sufficient data related to the effect of this fungicide, weedicide and algicides on soil nutrient availability, hence it needs to be studied in future.

The results revealed that the application of all the chemicals significantly reduced the beneficial soil microbe's viz., bacteria, fungi, actinomycetes, phosphobacteria, Azospirillum and Pseudomonas. This might be due to the inhibitory effect of chemicals on soil microbes (Table 4 and 5).

Ponnuswamy et al., (1997) reported that the application of various weedicides in soybean affected the bacterial population in soil. All the plots treated with weedicides gave depressing fungal population in first few days of application and regained its population after long period. Similar observations were recorded by Anonymous (1971) and Mukhopadhyay (1980).

Fungicides are used extensively in modern agriculture for the control of fungal pathogens. The chemicals alter the number 
and activity of microorganism and thus affect biochemical processes and fertility of the soil (Wainwright, 1978). Majority of biochemical transformation in soil results from microbial activity. Fungicides treated soil harboured less population of fungi in comparison to control. This is in conformity with earlier reports of several authors (Colinas et al., 1994; Sukla et al., 1987).

Investigations were carried out on the effects of $\mathrm{Cu}$ and other heavy metals on soil microorganisms, invariably indicated the adverse effects of these elements on the soil microbial activity, number and population as reported earlier by various workers (Olson and Thornton, 1982; Duxbury and Bicknell, 1983; Maliszewska et al., 1985; Hiroki, 1992; Chander and Brookes, 1991, 1993; Hattori, 1992; Huysman et al., 1994; Doelman and Haanstra, 1984; Aoyama and Nagumo, 1996; 1997). For example excessive heavy metal concentration in the soil has been reported to cause a decrease in microbial population (Hicks et al., 1990; McGrath et al., 1995), changes in populations structure (Chaudri et al., 1993; Bardgett et al., 1994; Huysman et al., 1994) and physiological activity (Bitton and Dutka, 1986; Cotrufo et al., 1995; Valsecchi et al., 1995).

Copper is known to be very toxic to microorganisms in the free ionic form especially $\mathrm{Cu}^{2+}$ and $\mathrm{CuOH}^{+}$(Zevenhuizen et al., 1979). The addition of copper to soil was reported to significantly decrease the amount of microbial biomass and exert a pronounce toxic effect on the size of biomass compared to certain metals such as $\mathrm{Pb}$ and $\mathrm{As}$ when compared on a molarity basis (Aoyama and Nagumo, 1997).

Effect of fungicide, weedicide and algicide application on plant growth parameters under field conditions

Application of $\mathrm{CuSO}_{4}$ significantly increases the root length $(22 \mathrm{~cm} /$ plant $)$, shoot length (108 cm/plant) and grain yield $(4962.5 \mathrm{~kg} / \mathrm{ha})$ than the other treatments (Table 6). Horii et al., (2007) reported that the germination percent of fungicide treated seeds got increased with increase in concentration of fungicide. Various other studies also found that seed germination was stimulated by thiamethoxam in soybean, pea and corn (Cataneo et al., 2010). The shoot length was increased with increase in concentration of fungicide. Windham \& Windham (2004) reported that systemic fungicides, which are based on sterol biosynthesis inhibitor, are closely related to plant growth regulators, the use of which at higher than labeled rates shorten the internodes which may lead to slow shoot growth. Vigor index of fungicide treated seedlings got increased with increase in concentration of fungicide. The increase in vigor index was significant at almost all the concentration of fungicide as compared to control. These present findings supports Doyle et al., (2001) proved that seedlings treated with thiamethoxam had a particular advantage of improved seedling vigor. Csinos (2004) revealed that mefenoxam improved vigor index of tobacco. All herbicidal treatments significantly increased yield and yield components like seed yield, test weight, pod dry weight, number of pods / plant, number of seeds / plant, harvest index (Amarogouda et al., 2013).

Copper is one of the essential micronutrients for plant growth and involved in numerous physiological functions as a component of several enzymes, mainly those which participate in electron flow, catalyze redox reactions in mitochondria and chloroplasts (Lolkema and Vooijs, 1986; Harrison et al., 1999; Hansch and Mendel, 2009). However, in large amount copper becomes toxic as it interferes with photosynthetic and respiratory processes, protein synthesis and development of plant organelles (Agarwala et al., 1995; Upadhyay and Panda, 2009). Specifically, 
excess copper can cause chlorosis, inhibition of root growth and damage to plasma membrane permeability, leading to ion leakage (Ouzounidou et al., 1992; Berglund et al., 2002; Bouazizi et al., 2010).

Pankaj Giri et al., (2014) findings suggest that
$\mathrm{Cu}$ fluxes affect the growth and yield of wheat plants. Application of $\mathrm{Cu}$ in low amount may promote growth, pigments synthesis, protein and sugar contents while in excess amount it adversely affects the yield and other growth parameters.

Table.1 The physicochemical and microbiological populations of the experimental field soil

\begin{tabular}{|c|c|}
\hline Soil properties & Mean $\pm \mathrm{SE}$ \\
\hline Soil type & Clay loam \\
\hline pH & $7.8 \pm 0.09$ \\
\hline $\mathrm{EC}\left(\mathrm{dSm}^{-1}\right)$ & $0.04 \pm 0.15$ \\
\hline Organic carbon (\%) & $0.50 \pm 0.01$ \\
\hline Available N (\%) & $162 \pm 2.88$ \\
\hline Available P (\%) & $35 \pm 0.57$ \\
\hline Available K (\%) & $250 \pm 1.45$ \\
\hline Total bacteria (cfu $\times 10^{5} / g$ drwt of soil) ${ }^{a}$ & $46 \pm 1.15$ \\
\hline Fungi (cfu $\times 10^{3} / g$ drwt of soil) ${ }^{b}$ & $2 \pm 0.16$ \\
\hline Diazotrophs (cfu $\times 10^{4} / g$ drwt of soil) ${ }^{c}$ & $21 \pm 1.15$ \\
\hline
\end{tabular}

Table.2 Effect of weedicides, fungicides and algicides application on algae growth under laboratory/field conditions

\begin{tabular}{|c|c|c|c|}
\hline Treatments & $\begin{array}{l}\text { Recommended } \\
\text { dose /ha }\end{array}$ & $\begin{array}{l}\text { Laboratory conditions } \\
\text { (dosage: } 2 \mathrm{ml} / \text { plate) }\end{array}$ & Field conditions \\
\hline$T_{1}$-Bispyriphos sodium & $20 \mathrm{~g}$ & No inhibition & No inhibition \\
\hline $\mathbf{T}_{2}-\mathbf{A l m i x}$ & $20 \mathrm{~g}$ & No inhibition & No inhibition \\
\hline $\mathbf{T}_{3}-$ Pyrosulfuran & $200 \mathrm{~g}$ & No inhibition & No inhibition \\
\hline$T_{4}$ - Londox power & $10 \mathrm{Kg}$ & Moderate inhibition & Moderate inhibition \\
\hline $\mathrm{T}_{5}-\mathrm{CuSO}_{4}$ & $2.5 \mathrm{Kg}$ & Moderate inhibition & Inhibition \\
\hline $\mathrm{T}_{6}-\mathrm{CaO}$ & $25 \mathrm{Kg}$ & No inhibition & No inhibition \\
\hline $\mathrm{T}_{7}-\mathrm{CuSO}_{4}+\mathrm{CaO}$ & $2.5 \mathrm{~kg}+25 \mathrm{Kg}$ & Moderate inhibition & Moderate inhibition \\
\hline $\mathbf{T}_{8}$-Butachlor & 2.5 lit & No inhibition & No inhibition \\
\hline $\mathbf{T}_{9}$-Propiconazole & $250 \mathrm{ml}$ & Moderate inhibition & No inhibition \\
\hline$T_{10}$-Hexaconazole & $250 \mathrm{ml}$ & Moderate inhibition & No inhibition \\
\hline $\mathbf{T}_{11}-$ control & - & No inhibition & No inhibition \\
\hline
\end{tabular}


Table.3 Effect of weedicides, fungicides and algicides application on available N, P and K content of the soil infected with green algae under wet land rice (ADT 43) ecosystem

\begin{tabular}{|c|c|c|c|}
\hline Treatments & $\begin{array}{c}\text { Available N } \\
\text { (kg/ha) }\end{array}$ & $\begin{array}{c}\text { Available P } \\
\text { (kg/ha) }\end{array}$ & $\begin{array}{c}\text { Available K } \\
\text { (kg/ha) }\end{array}$ \\
\hline $\mathrm{T}_{1}$-Bispyriphossodium & 831.25 & 75 & 775.0 \\
\hline $\mathbf{T}_{2}-$ Almix & 385.0 & 58 & 462.5 \\
\hline$T_{3}-$ Pyrosulfuran & 317.5 & 117 & 287.5 \\
\hline $\mathbf{T}_{4}$ - Londox power & 332.5 & 95 & 400.0 \\
\hline $\mathrm{T}_{5}-\mathrm{CuSO}_{4}$ & 280.0 & 64 & 437.5 \\
\hline $\mathrm{T}_{6}-\mathrm{CaO}$ & 202.5 & 104 & 262.5 \\
\hline $\mathrm{T}_{7}-\mathrm{CuSO}_{4}+\mathrm{CaO}$ & 177.5 & 142 & 350.0 \\
\hline $\mathbf{T}_{8}-$ Butachlor & 650.0 & 181 & 237.5 \\
\hline $\mathbf{T}_{9}$-Propiconazole & 332.5 & 72 & 512.5 \\
\hline $\mathbf{T}_{10}$-Hexaconazole & 280.0 & 83 & 225.0 \\
\hline$T_{11}$-control & 260.0 & 34 & 400.0 \\
\hline SEd & 26.13 & 6.25 & 25.69 \\
\hline $\mathrm{CD}(\mathrm{P}=0.05)$ & 54.89 & 13.15 & 53.97 \\
\hline
\end{tabular}

Table.4 Effect of weedicides, fungicides and algicides application on microbial population in the rhizosphere of soil cropped with rice (ADT 43) under wet land rice ecosystem

\begin{tabular}{|c|c|c|c|c|c|c|c|c|c|}
\hline \multirow[t]{2}{*}{ Treatments } & \multicolumn{3}{|c|}{$\begin{array}{c}\text { Bacteria } \\
\left(\times 10^{4} \mathrm{cfu} / \mathrm{g}\right)\end{array}$} & \multicolumn{3}{|c|}{$\begin{array}{c}\text { Fungi } \\
\left(x 1^{3} \mathrm{cfu} / \mathrm{g}\right)\end{array}$} & \multicolumn{3}{|c|}{$\begin{array}{l}\text { Actinomycetes } \\
\text { (x 10²fu/g) }\end{array}$} \\
\hline & $\begin{array}{c}30 \\
\text { DAP }\end{array}$ & $\begin{array}{c}60 \\
\text { DAP }\end{array}$ & $\begin{array}{c}90 \\
\text { DAP }\end{array}$ & $\begin{array}{c}30 \\
\text { DAP }\end{array}$ & $\begin{array}{c}60 \\
\text { DAP }\end{array}$ & $\begin{array}{c}90 \\
\text { DAP }\end{array}$ & $\begin{array}{c}30 \\
\text { DAP }\end{array}$ & $\begin{array}{c}60 \\
\text { DAP }\end{array}$ & $\begin{array}{c}90 \\
\text { DAP }\end{array}$ \\
\hline$T_{1}$ - Bispyriphos sodium & 27 & 15 & 8 & 5 & 3 & 0 & 24 & 15 & 7 \\
\hline$T_{2}-$ Almix & 34 & 28 & 20 & 20 & 12 & 5 & 18 & 12 & 5 \\
\hline$T_{3}$-Pyrosulfuran & 30 & 24 & 12 & 12 & 8 & 3 & 13 & 9 & 4 \\
\hline $\mathrm{T}_{4}$ - Londox power & 32 & 27 & 20 & 22 & 15 & 7 & 10 & 7 & 5 \\
\hline $\mathrm{T}_{5}-\mathrm{CuSO}_{4}$ & 40 & 32 & 15 & 27 & 17 & 11 & 27 & 19 & 8 \\
\hline $\mathrm{T}_{6}-\mathrm{CaO}$ & 32 & 25 & 17 & 25 & 15 & 8 & 25 & 15 & 6 \\
\hline $\mathrm{T}_{7}-\mathrm{CuSO}_{4}+\mathrm{CaO}$ & 35 & 28 & 22 & 18 & 12 & 4 & 27 & 18 & 12 \\
\hline $\mathbf{T}_{8}$-Butachlor & 34 & 26 & 15 & 25 & 16 & 9 & 23 & 14 & 11 \\
\hline$T_{9}$-Propiconaole & 28 & 17 & 8 & 22 & 14 & 6 & 21 & 11 & 7 \\
\hline$T_{10}$-Hexaconazole & 32 & 23 & 11 & 20 & 12 & 7 & 19 & 8 & 6 \\
\hline SEd & 1.93 & 1.49 & 0.93 & 1.23 & 0.79 & 0.41 & 1.20 & 0.75 & 0.44 \\
\hline $\mathrm{CD}(\mathrm{P}=\mathbf{0 . 0 5})$ & 4.06 & 3.13 & 1.97 & 2.59 & 1.66 & 0.85 & 2.53 & 1.59 & 0.92 \\
\hline
\end{tabular}


Table.5 Effect of weedicides, fungicides and algicides on beneficial microbial population in the rhizosphere of soil cropped with rice (ADT 43) under wet land rice ecosystem

\begin{tabular}{|c|c|c|c|c|c|c|c|c|c|}
\hline \multirow[b]{2}{*}{ Treatments } & \multicolumn{3}{|c|}{$\begin{array}{l}\text { Phosphobacteria } \\
\left.\text { (x 10 } 10^{4} \mathrm{cfu} / \mathrm{g}\right)\end{array}$} & \multicolumn{3}{|c|}{$\begin{array}{l}\text { Azospirillum } \\
\left(\times 10^{5} \mathrm{MPN} / \mathrm{g}\right)\end{array}$} & \multicolumn{3}{|c|}{$\begin{array}{c}\text { Pseudomonas } \\
\left(\times 10^{4} \mathrm{cfu} / \mathrm{g}\right)\end{array}$} \\
\hline & $\begin{array}{c}30 \\
\text { DAP }\end{array}$ & $\begin{array}{c}60 \\
\text { DAP }\end{array}$ & $\begin{array}{c}90 \\
\text { DAP }\end{array}$ & $\begin{array}{c}30 \\
\text { DAP }\end{array}$ & $\begin{array}{c}60 \\
\text { DAP }\end{array}$ & $\begin{array}{c}90 \\
\text { DAP }\end{array}$ & $\begin{array}{c}30 \\
\text { DAP }\end{array}$ & $\begin{array}{c}60 \\
\text { DAP }\end{array}$ & $\begin{array}{c}90 \\
\text { DAP }\end{array}$ \\
\hline$T_{1}$ - Bispyriphos sodium & 15 & 10 & 4 & 1.2 & 0.9 & 1.0 & 18 & 7 & 5 \\
\hline $\mathbf{T}_{2}-\mathbf{A}$ Imix & 25 & 18 & 10 & 1.4 & 0.8 & 0.1 & 25 & 20 & 18 \\
\hline$T_{3}$-Pyrosulfuran & 18 & 12 & 6 & 2.5 & 1.4 & 0.5 & 25 & 20 & 12 \\
\hline$T_{4}$ - Londox power & 26 & 20 & 10 & 2.5 & 1.8 & 0.6 & 23 & 18 & 11 \\
\hline $\mathrm{T}_{5}-\mathrm{CuSO}_{4}$ & 30 & 25 & 12 & 2.8 & 1.8 & 0.9 & 40 & 32 & 22 \\
\hline $\mathrm{T}_{6}-\mathrm{CaO}$ & 28 & 20 & 12 & 2.2 & 1.5 & 0.1 & 30 & 25 & 16 \\
\hline $\mathrm{T}_{7}-\mathrm{CuSO}_{4}+\mathrm{CaO}$ & 27 & 20 & 12 & 1.4 & 0.8 & 0.1 & 36 & 30 & 22 \\
\hline$T_{8}$-Butachlor & 20 & 14 & 7 & 2.5 & 1.6 & 0.6 & 35 & 28 & 15 \\
\hline$T_{9}$-Propiconaole & 17 & 12 & 6 & 1.8 & 1.5 & 1.0 & 24 & 22 & 11 \\
\hline $\mathrm{T}_{10}$-Hexaconazole & 12 & 6 & 2 & 1.4 & 0.8 & 0.5 & 20 & 15 & 8 \\
\hline SEd & 1.35 & 1.02 & 0.52 & 0.12 & 0.08 & 0.042 & 1.66 & 1.33 & 0.86 \\
\hline $\mathrm{CD}(\mathrm{P}=\mathbf{0 . 0 5})$ & 2.84 & 2.14 & 1.11 & 0.26 & 0.017 & 0.087 & 3.49 & 2.80 & 1.81 \\
\hline
\end{tabular}

Table.6 Effect of the fungicides, weedicides and algicides application on root length, shoot length and grain yield of rice (ADT 43) under wet land rice ecosystem

\begin{tabular}{|c|c|c|c|c|c|c|c|}
\hline \multirow[b]{2}{*}{ Treatments } & \multicolumn{3}{|c|}{$\begin{array}{l}\text { Root length } \\
\text { (cm/plant) }\end{array}$} & \multicolumn{3}{|c|}{$\begin{array}{c}\text { Shoot length } \\
\text { (cm/plant) }\end{array}$} & \multirow{2}{*}{$\begin{array}{c}\text { Yield } \\
(\mathrm{Kg} / \mathrm{ha})\end{array}$} \\
\hline & $\begin{array}{c}30 \\
\text { DAS }\end{array}$ & $\begin{array}{c}60 \\
\text { DAS }\end{array}$ & $\begin{array}{c}90 \\
\text { DAS }\end{array}$ & $\begin{array}{c}30 \\
\text { DAS }\end{array}$ & $\begin{array}{c}60 \\
\text { DAS }\end{array}$ & $\begin{array}{c}90 \\
\text { DAS }\end{array}$ & \\
\hline$T_{1}-$ Bispyriphos sodium & 11.0 & 19 & 9.0 & 53.5 & 73.5 & 93.5 & 3568.0 \\
\hline $\mathbf{T}_{2}-\mathbf{A}$ Imix & 12.5 & 17.0 & 8.0 & 54.0 & 74.0 & 94.0 & 3913.0 \\
\hline$T_{3}$-Pyrosulfuran & 14.0 & 18.0 & 10 & 52.5 & 72.5 & 92.5 & 4887.5 \\
\hline$T_{4}$ - Londox power & 12.0 & 16.0 & 8.0 & 53.5 & 73.5 & 93.5 & 3762.5 \\
\hline $\mathrm{T}_{5}-\mathrm{CuSO}_{4}$ & 18.0 & 22.0 & 14 & 68.0 & 88.0 & 108.0 & 4962.5 \\
\hline $\mathrm{T}_{6}-\mathrm{CaO}$ & 15.0 & 19.5 & 11 & 52.5 & 72.5 & 92.5 & 4837.5 \\
\hline $\mathrm{T}_{7}-\mathrm{CuSO}_{4}+\mathrm{CaO}$ & 16.5 & 21.0 & 11 & 61.5 & 81.5 & 101.5 & 4487.5 \\
\hline $\mathbf{T}_{8}$-Butachlor & 14.0 & 17.0 & 8.0 & 60.5 & 80.5 & 100.5 & 4775.0 \\
\hline$T_{9}$-Propiconazole & 13.0 & 18.0 & 9.0 & 57.5 & 77.5 & 97.5 & 3468.0 \\
\hline$T_{10}$-Hexaconazole & 11.0 & 16.0 & 8.0 & 48.5 & 68.5 & 92.5 & 4712.5 \\
\hline SEd & 0.81 & 1.08 & 0.57 & 3.35 & 4.54 & 5.75 & 3.807 \\
\hline $\mathrm{CD}(\mathrm{P}=0.05)$ & 1.71 & 2.27 & 1.21 & 7.05 & 9.54 & 12.089 & 7.999 \\
\hline
\end{tabular}


Table.7 Physical parameters of the soil sample at Maharajapuram field

\begin{tabular}{|c|c|c|c|c|c|}
\hline $\begin{array}{c}\text { Date of } \\
\text { sampling }\end{array}$ & $\begin{array}{c}\mathbf{E C} \\
\left(\mathbf{d S m}^{-\mathbf{1}}\right)\end{array}$ & $\mathbf{p H}$ & $\begin{array}{c}\mathbf{N} \\
\mathbf{( K g / h a )}\end{array}$ & $\begin{array}{c}\mathbf{P} \\
(\mathbf{K g} / \mathbf{h a})\end{array}$ & $\begin{array}{c}\mathbf{K} \\
(\mathbf{K g} / \mathbf{h a})\end{array}$ \\
\hline $\mathbf{1 6 . 1 0 . 1 5}$ & 0.63 & 8.2 & 63.0 & 25 & 90 \\
\hline $\mathbf{0 5 . 1 1 . 1 5}$ & 0.16 & 8.1 & 32.2 & 50 & 75 \\
\hline $\mathbf{2 5 . 1 1 . 1 5}$ & 0.14 & 8.0 & 46.2 & 38 & 155 \\
\hline
\end{tabular}

Table.8 Effect of $\mathrm{CuSO}_{4}$ application on algae growth under field conditions

\begin{tabular}{|c|c|c|c|}
\hline Treatments & Dosage/ha & $\begin{array}{c}\text { Aduthuraifield } \\
\text { condition }\end{array}$ & $\begin{array}{l}\text { Maharajapuram } \\
\text { field condition }\end{array}$ \\
\hline $\begin{array}{l}\mathrm{T}_{1} \text {-Alternate wetting } \\
\text { and thawing the } \\
\text { algal growth with } \\
\text { conoweeder if algal } \\
\text { growth occurred }\end{array}$ & - & Inhibition & Moderate inhibition \\
\hline $\mathrm{T}_{2}-\mathrm{CuSO}_{4}$ & Soil application- $1 \mathrm{Kg}$ & Inhibition & Moderate inhibition \\
\hline $\mathbf{T}_{3}-\mathbf{T}_{1}+\mathbf{T}_{2}$ & $\begin{array}{c}\text { As indicated } \\
\text { Above }\end{array}$ & Inhibition & Moderate inhibition \\
\hline $\mathrm{T}_{4}-\mathrm{CuSO}_{4}$ & Soil drenching- $0.5 \%$ & Moderate inhibition & Moderate inhibition \\
\hline $\mathrm{T}_{5}-\mathrm{Cu}(\mathrm{OH})_{2}$ & Soil application $-1 \mathrm{Kg}$ & Moderate inhibition & No inhibition \\
\hline$T_{6}-T_{1}+T_{5}$ & $\begin{array}{c}\text { As indicated } \\
\text { Above }\end{array}$ & Inhibition & No inhibition \\
\hline $\mathrm{T}_{7} \cdot \mathrm{Cu}(\mathrm{OH})_{2}$ & Soil Drencing-0.5\% & Moderate inhibition & No inhibition \\
\hline$T_{8}$-Londox power & Soil application $-1 \mathrm{Kg}$ & Moderate inhibition & No inhibition \\
\hline$T_{9}-T_{1}+T_{8}$ & $\begin{array}{l}\text { As indicated } \\
\text { Above }\end{array}$ & Inhibition & No inhibition \\
\hline $\mathbf{T}_{10}$-Londox power & Soil drenching $-0.5 \%$ & Moderate inhibition & No inhibition \\
\hline $\begin{array}{l}\mathrm{T}_{11} \text {-Soil extract with } \\
\text { cell free extract } \\
\text { containing } \\
\text { Rhamnolipids from } \\
\text { Pseudomonas } \\
\text { chlororamphis }\end{array}$ & $10 \%$ & No inhibition & No inhibition \\
\hline $\mathbf{T}_{12}$-Control & - & No inhibition & No inhibition \\
\hline
\end{tabular}

Table.9 Physical parameters of the water sample at Maharajapuram field

\begin{tabular}{|c|c|c|c|c|c|c|c|c|c|}
\hline $\begin{array}{c}\text { Date of } \\
\text { sampling }\end{array}$ & $\mathbf{p H}$ & $\begin{array}{c}\mathbf{E C} \\
\left(\mathbf{d S m}^{-1}\right)\end{array}$ & $\begin{array}{c}\mathbf{H C O} \\
(\mathbf{p p m})\end{array}$ & $\begin{array}{c}\mathbf{S} \\
(\mathbf{p p m})\end{array}$ & $\begin{array}{c}\mathbf{C l} \\
(\mathbf{p p m})\end{array}$ & $\begin{array}{c}\mathbf{C a} \\
(\mathbf{p p m})\end{array}$ & $\begin{array}{c}\mathbf{M g} \\
(\mathbf{p p m})\end{array}$ & $\begin{array}{c}\mathbf{N a} \\
(\mathbf{p p m})\end{array}$ & $\begin{array}{c}\mathbf{K} \\
(\mathbf{p p m})\end{array}$ \\
\hline $\mathbf{1 6 . 1 0 . 1 5}$ & 8.2 & 1.40 & 5.2 & 0.138 & 7.4 & 3.4 & 4.5 & 5.2 & 0.1 \\
\hline $\mathbf{0 5 . 1 1 . 1 5}$ & 8.0 & 1.38 & 4.8 & 0.125 & 7.1 & 3.1 & 4.2 & 5.4 & 0.1 \\
\hline $\mathbf{2 5 . 1 1 . 1 5}$ & 7.9 & 1.11 & 4.4 & 0.63 & 4.7 & 2.9 & 2.8 & 5.0 & 0.1 \\
\hline
\end{tabular}


Fig.1 Green algae infected field
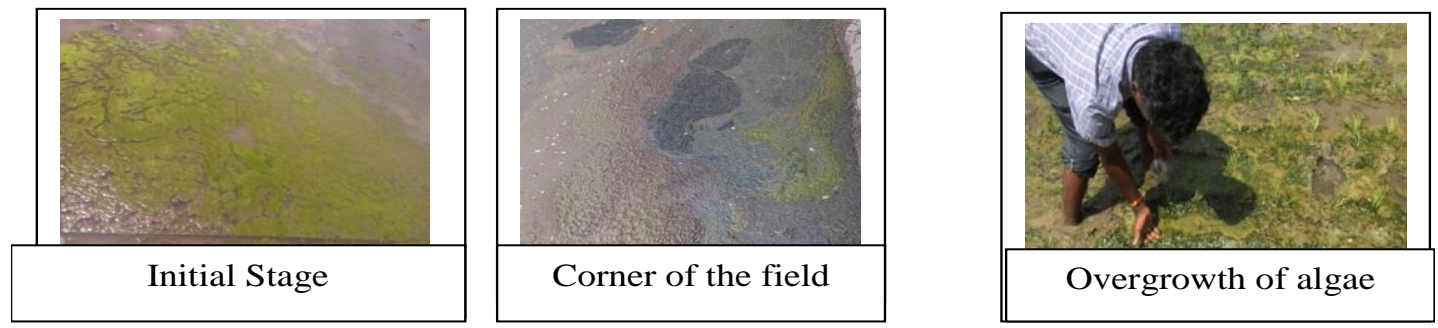

Fig.2 Overview of the field experiment
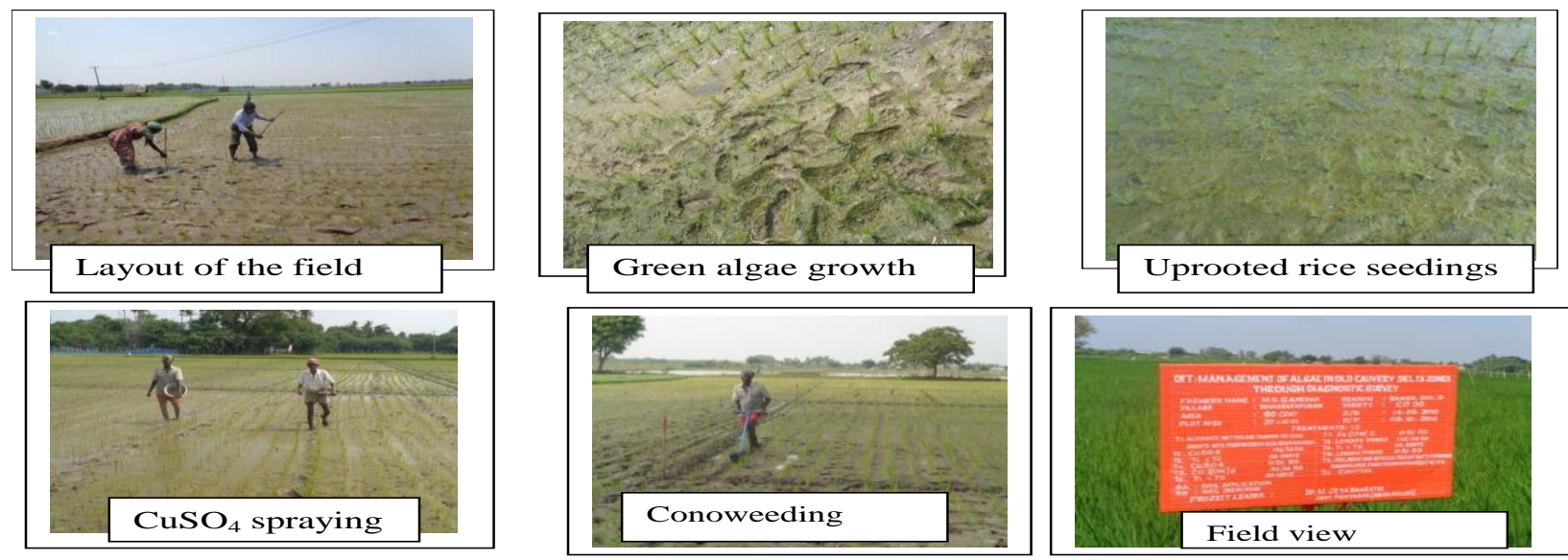

\section{Graphical Abstract}
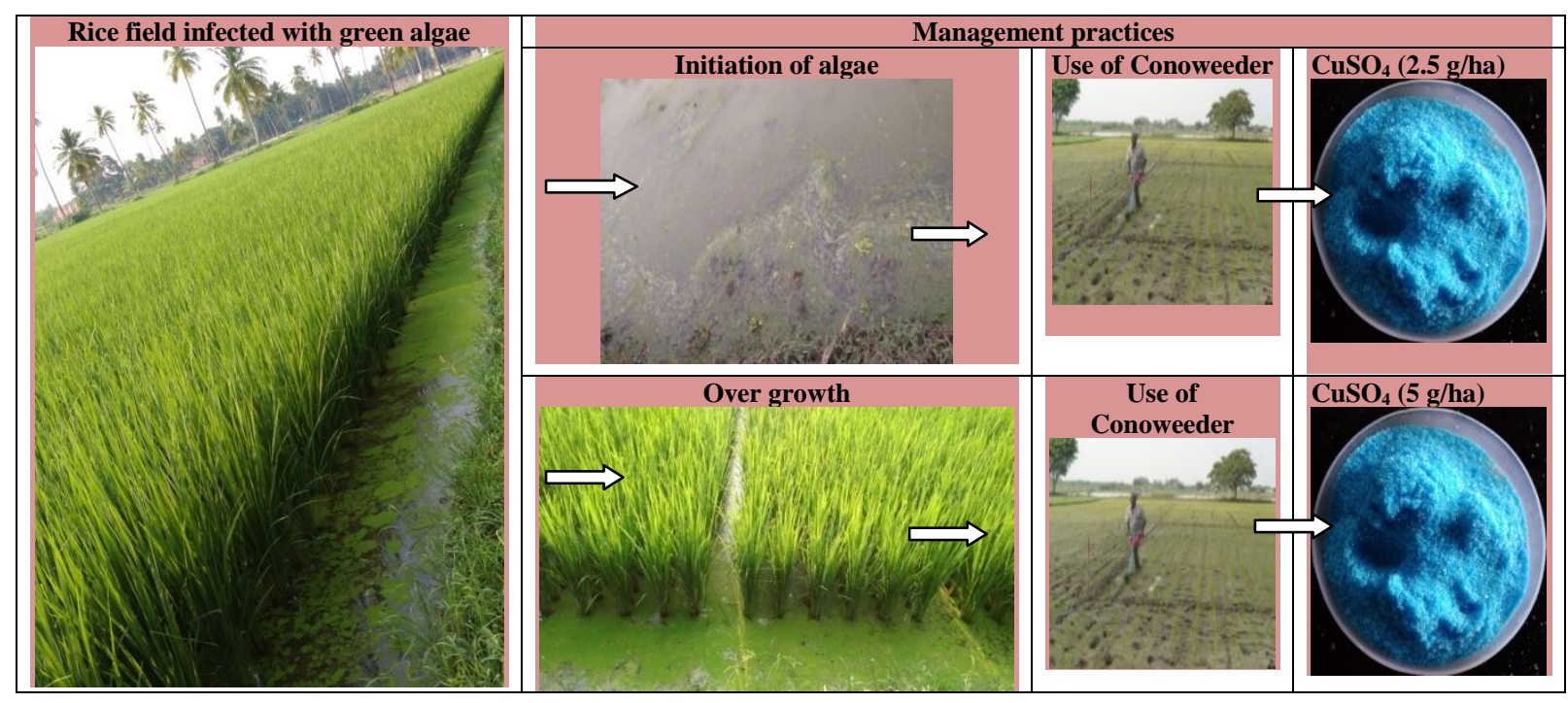


\section{Effect of $\mathrm{CuSO}_{4}$ application on green algae growth at Aduthurai}

The results of the confirmatory field experiment at Aduthurai revealed that by simple physical management practices like alternate wetting and drying, manual trampling of algae and use of conoweeder reduces the green algae growth (Table 6). Repeated stagnation of bore well water increases the phosphorus content in soil leads to the stimulation of algae spore. When stimulation of algae spores observed, water was completely drained, $\mathrm{CuSO}_{4}$ drenching at the rate of $2.5 \mathrm{~kg} / \mathrm{ha}$ was done. But during tremendous algae growth, $\mathrm{CuSO}_{4}$ drenching at the rate of $5.0 \mathrm{~kg} / \mathrm{ha}$ was done. In most cases action to eradicate green algae is taken only when filaments are present in huge numbers and the treatments available are limited. Generally Australian farmers use water control, bluestone $\left(\mathrm{CuSO}_{4}\right)$ and coptrol (2. 5 lit/ha), both of which are drastic answers to a problem where a lot of damage has already been done (Hrudey et al., 1999, Jones and Burch, 1997; Jones and Orr, 1994 and http//www.affa.gov.au/nra/welcome.html accessed January 2002).

PAK $^{\text {TM} 27 ~(B i o S a f e ~ S y s t e m s, ~} 2008$ and Hazardous Substances Data Bank, 2009) is a granular product that attacks planktonic and filamentous algae on contact. The active ingredient is sodium carbonate peroxyhydrate and creates a powerful oxidation reaction that destroys algal cell membranes and chlorophyll providing immediate control of algae. Fast acting within 60 seconds of application and leaves behind no harmful residues and adds 13\% bio-available oxygen to the water.

\section{Effect of $\mathrm{CuSO}_{4}$ application at Maharajapuram rice field}

The results of confirmatory field experiment at Maharajapuram showed that the algae growth was not completely arrested even after $3^{\text {rd }}$ day of $\mathrm{CuSO}_{4}$ application. Hence, the soil and water sample were collected from the Maharajapuram field to know the physical parameters of the soil. Maharajapuram soil and water consists of alkaline $\mathrm{pH}$ (8.2) and more $\mathrm{HCO}_{3}$ ions (5.2). This is the main cause of overgrowth of green algae which was not controlled by recommended quantity ${ }_{\text {ofCuSO}} \quad(5 \mathrm{~kg} / \mathrm{ha})$ application. This was supported by the following findings. In culture media, the optimal $\mathrm{pH}$ for the growth of cyanobacteria ranges from $7.5-10$, with a lower limit of $6.5-7.0$. However, in soil culture experiments, soils having slightly alkaline reaction were more favourable, while in natural environments cyanobacteria prefer neutral to alkaline $\mathrm{pH}$ (De, 1939 and Roger and Reynaud, 1979).

In this regard $\mathrm{pH}$ is one of most important factor which affects the presence of BGA in a habitat. Saline and Sodic (alkali) nature of soils significantly reduce the value and productivity of affected lands. Salt affected soil are divided into three groups depending on the total soluble salts (measured in the terms of Electrical Conductivity E. C.), soil $\mathrm{pH}$ and Exchangeable $\mathrm{Na} \%$. Usar soils are grouped into two divisions Saline (Solanchak) and Alkalines (Solonetz). (Pandey et al., 2005)

It is concluded that soil is consider as a buffer that can tolerate the $\mathrm{pH}$ change. Even though continuous dumping of DAP and other chemical fertilizers changes the soil $\mathrm{pH}$, innate capacity and fix more amount of the salt in the soil, which indeed difficult by reclamation practices $\left(\mathrm{CuSO}_{4}\right)$. In this situation continuous treatments like gypsum (bring the $\mathrm{pH}$ in acidic), infiltration with water (leach excess ions viz. $\mathrm{Na}, \mathrm{HCO}_{3}$ ), biofertilizers treatment (to move the accumulated P) and periodical soil and water sample analysis are important. It is always 
advisable to prevent the excess algae growth by fertilizer and water management practices rather than control, since chemicals are toxic to soil microorganisms. However, when situation warrants, application of $2.5 \mathrm{~kg}$ of $\mathrm{CuSO}_{4} / \mathrm{ha}$ when the growth of algae is mild and $5.0 \mathrm{~kg} / \mathrm{ha}$ when severe algae growth is noticed is the best management practices.

\section{Acknowledgement}

I would like to thank Dr. V. Ravi, Director, Tamil Nadu Rice Research Institute, Aduthurai, Tamil Nadu for the Initiative spirit, assistantship and guidance to work in the area.

\section{References}

Agarwala SC, Nautiyal BD, Chatterjee C and Nautiyal N. 1995. Variations in copper and zinc supply influence growth and activities of some enzymes in maize. Soil Science and Plant Nutrtion,41: 329-335.

Allen DN. 1953. Experiments in Soil bacteriology (IIed.) Burgees Publ. Co., Indian Journal of Microbiology, 32: 469 -472.

Amaregouda A, Jitendra Jadhav, Chetti MB and Nawalagatti. 2013. Effect of weedicide on Physiological parameters, Growth, yield and yield components of soybean (Glycine max. L) and weed growth. Journal of Agriculture and Allied Sciences, 4(2): 12 15

Anonymous. 1971. Report of biological nitrogen fixation scheme. Mahatma Phpule Krishi Vidyapeeth, Rahuri (AGRISCO).

Aoyama M and Nagumo T. 1996. Factors affecting microbial biomass and dehydrogenase activity in apple orchard soils with heavy metal accumulation. Soil Science and Plant Nutrition, 42: 821-831.

Aoyama, M and Nagumo T. 1997. Comparison of the effects of $\mathrm{Cu}, \mathrm{Pb}$ and as on plant residue decomposition, microbial biomass and soil respiration. Soil Science and Plant Nutrition, 43: 613-622.

Bardgett, RD, Speir TW, Ross DJ, Yeates GW and Kettles HA. 1994. Impact of pasture contamination by copper, chromium and arsenic timber preservative on soil microbial properties and nematodes. Journal of Biology and Fertility of Soils, 18: 71-79.

Bargali, SS., Bargali, K., Singh, L., Ghosh, L. and Lakhera, M.L. 2009. Acacia niloticabased traditional agroforestry system: effect on paddy crop and management. Curent Science 96(4): 581-587.

Bargali, SS., Singh, SP., Shrivastava, SK. and Kolhe, SS. 2007. Forestry plantations on rice bunds: Farmers' perceptions and technology adoption. International Rice Research Notes 32(2): 40-41.

Berglund AH, Mike F, Quartacci MF, Calucci LC, Navari-Izzo F, Pinzino C. and Liljenberg C. 2002. Alterations of wheat root plasma membrane lipid composition induced by copper stress result in changed physicochemical properties of plasma membrane lipid vesicles. Biochimica et Biophysica Acta, 1564: 466-472.

Biosafe Systems. 2008. Green Clean Pro: Granular Algaecide/Fungicide. Specimen Label. BioSafe Systems, LLC, Connecticut.

Bitton, G and Dutka BJ. 1986. Toxicity Testing using Microbes, Volume 1. CRC Press, Baca Raton, FL.

Bouazizi H, Jouili H, Geitmann A. and Ferjani EEI. 2010. Copper toxicity in expanding leaves of Phaseolus vulgaris L.: antioxidant enzyme response and nutrient element uptake. Eco toxically and Environmental Safety, 73: 1304-1308 (2010).

Cataneo, AC, Ferreira LC, Carvalho JC, AndréoSouza Y, Corniani N, Mischan MM, Nunes JC. 2010. Improved germination of soybean seed treated with thiamethoxam under drought conditions. Seed Science and Technology,38:248-251.

Chander, K., and Brookes, PC. 1991. Is the dehydrogenase assay invalid as a method to estimate microbial activity in copper contaminated soil. Soil Biology and Biochemistry, 23: 909-915.

Chander, K., and Brookes, PC. 1993. Residual effects of zinc, copper and nickel in sewage sludge on microbial biomass in a 
sandy loam. Soil Biology and Biochemistry, 25: 1231-1239

Chaudri, AM, McGrath SP, Giller KE, Rietz E. and Sauerbeck DR. 1993. Enumeration of indigenous Rhizobium leguminosarum biovar trifoliiin soils previously treated with metal-contaminated sewage sludge. Soil Biology and Biochemistry, 25: 301309.

Colinas, C, Ingham E. and Molina R. 1994. Population response of target and non target forest soil organisms to selected biocides. Soil Biology and Biochemistry, 26: $41-47$

Coptrol, Rural chemicals industries (Australia) private limited, 1957. Australia Common wealth Trading Bank of Australia Ltd, Castle Towers, Shopping Centre, Castle Hill, NSW 2154

Cotrufo, MF, de Santo AV, Alfani A, Battoli G. and de Grisrofaro. 1995. Effects of urban heavy metal pollution on organic matter decomposition in Quercus ilex L. woods. Environmental Pollution, 89: 81-87.

Csinos, AS. 2004. Efficacy of fungicides on tobacco blank shank. Phytopathology. 94:9.

De, PK. 1939. The role of blue-green algae in nitrogen fixation in rice fields. Proceedings of the Royal Society of London Series B 127: 121-139.

Doelman, P and Haanstra L. 1984. Short-term and long-term effects of cadmium, chromium, copper, nickel, lead and zinc on soil microbial respiration in relation to abiotic soil factors. Plant Soil, 79: 317-327.

Doyle P, Stypa M, Schneidersmann F and Ramachandran R. 2001. New generation seed treatment products for canola (Brassica napus, B. campestris) and mustard (Sinapsis alba, Brassica juncea). British crop protaction council Symposium proceedings, 76:173-180.

Doyle P, Stypa M, Schneidersmann F and Ramachandran R. 2001. New generation seed treatment products for canola (Brassica napus, B. campestris) and mustard (Sinapsis alba, Brassica juncea).British crop protaction council Symposium proceedings. 76:173-180.

Duxbury T and Bicknell B. 1983. Metal-tolerant bacterial populations from natural and metal-polluted soils. Soil Biology and Biochemistry, 15: 243-250.

Hansch R and Mendel RR. 2009. Physiological functions of mineral micronutrients $(\mathrm{Cu}$, $\mathrm{Cu}, \mathrm{Mn}, \mathrm{Fe}, \mathrm{Ni}, \mathrm{Mo}, \mathrm{B}, \mathrm{Cl})$. Current Opinion in Plant Biology, 12: 259-266.

Harrison MD, Jones CE and Dameron CT. 1999. Copper chaperones: function structure and copper-binding properties. Journal of biological inorganic chemistry, 4: 145153.

Hattori, H. 1992. Influence of heavy metals on soil microbial activities. Soil Science and Plant Nutrition, 38: 93-100.

Hicks RJ, Stotzky G and Voris PV. 1990. Review and evaluation of the effects of xenobiotic chemicals on microorganisms in soil. Advances in Applied Microbiology, 35: 195-253.

Hiroki M. 1992. Effects of heavy metal contamination on soil microbial population. Soil Science and Plant Nutrition, 38: 141-147. 909

Hoek C. Van den, Mann D.G and Jahns H.M. 1995. Algae An introduction to phycology. Cambridge University Press, Cambridge. ISBN 0 -521 -30419 -9.

Horii A, McCue P and Shetty K. 2007. Enhancement of seed vigour following insecticide and phenolic elicitor treatment. Bioresource Technology, 98:623-632

Hrudey S, Burch M. Drikas M and Gregory R. 1999. Remedial Measures. Ch 9, pp 275312 in I. Chorus and J. Bartram (Eds). Toxic Cyanobacteria in Water. A guide to their public health consequences, monitoring and management. E\&FN Spon, London.

HSDB (Hazardous Substances Data Bank). 2009. TOXNET (Toxicology Data Network). National Library of Medicine. http://toxnet.nlm.nih.gov/cgibin/sis/search.

Huysman F, Verstraete W and Brookes PC. 1994. Effect of manuring practices and increased copper concentrations on soil microbial populations. Soil Biology and Biochemistry, 26: 103-110

James, N. 1958. Soil extract in Soil Microbiology. Canadian Journal of Microbiology, 4: 
363.

Jeffrey, D. Palmer, Douglas E, Soltis and Mark

W. Chase. 2004. The plant tree of life: an overview and some points of view. American Journal of Botany. 91 (10): 1437-1445

Jones, $G$ and Burch M. 1997. Algicide and Algistat Use in Australia. Occasional Paper, ARMCANZ Sub- Committee on Water Resources, Canberra.

Jones G. and Orr P. 1994. Release and degradation of microcystin following algicide treatment of a Microcystis aeruginosa bloom in a recreational lake, as determined by HPLC and protein phosphatase inhibition assay. Water Research, 28(4): 871-876.

Judd WS, Campbell CS, Kellogg EA, Stevens PF and Donoghue M.J. Sinauer. 2002. Plant systematics, a phylogenetic approach. Associates Inc., Sunderland Mass. ISBN 0-87893 -403-0, p156.

King KO, Ward MK and Raney DE. 1954. Two simple media for the demonstration of pyocyanin and fluorescein. Journal of Laboratory and Clinical Medicine,44: 301-307.

Lolkema PC and Vooijs R. 1986. Copper tolerance in Silenecucubalus: Subcellular distribution of copper and its effects on chloroplast and plastocyanin synthesis. Planta, 167: 30-36.

Maliszewska W, Dec S, Wierzbicka H. and Wozniakowska A. 1985. The influence of various heavy metal compounds on the development and activity of soil microorganisms. Environmental Pollution. Series A, Ecology and Biology, 37: 195215.

Martin JP. 1950. Use of acid, rose Bengal and streptomycin in the plate method for estimating soil fungi. Soil Science, 69: 215.

McGrath, SP, Chaudri AM and Giller KE. 1995. Long-term effects of metals in sewage sludge on soils, microorganisms and plants. Indian Journal of Microbiology, 14: 94-104.

Mukhopaddhay, SK. 1980. Effects of herbicides and Insecticides alone and their combinations on soil microflora. Indian
Journal of Weed Science, 12: 53-60.

National Registration Authority for Agricultural and Veterinary Chemicals, 1996. The Requirements Manual for Agricultural Chemicals. NRA, Canberra. NRA Website. www.affa.gov.au/nra/welcome.html

Okon Y, Albrect SL and Burris RH. 1977. Carbon and ammonia metabolism of Spirillum lipoferum. Journal of Bacteriology, 128: $592-597$.

Olsen SR, Cole CV, Watanabe FS and Dean L. 1954. Estimation of available phosphorus in soils by extraction with sodium bicarbonate. U.SDA. U.S. Govt. Printing Office, Washington, D.C. p. 989.

Olson BH and Thornton I. 1982. The resistance patterns to metals of bacterial populations in contaminated land. European Journal of Soil Science, 33: 271-277.

Ouzounidou G, Eleftheriou EP and Karataglis S. 1992. Ecophysiological and ultrastructural effects of copper in Thlaspiochroleucum (Curciferae). Canadian Journal of Botany, 70: 947-957.

Pandey KD, Shukla PN, Giri DD, Kashyap AK. 2005. Cyanobacteria in alkaline soil and the effect of cyanobacteria inoculation with pyrite amendments on their reclamation. Biology and Fertility of Soils, 41: $451-457$.

Pankaj Giri, Ram kumar, Rajeew Singh and Pandey SN. 2014. Effect of Cu application on growth, yield and biochemical responses of wheat (Triticum aestivum L.) in Gomti -upland alluvial soil. Research in Environment and Life Science, 7 (3): 201 204

Parkinson D, Gray JRG and Williams ST. 1971. In: Methods for studying the Ecology of soil Microorganisms. Oxford Blackwell scientific publications. P. 116.

Ponnuswamy P, Santhi G, Gopalswany and Mohamedali A. 1997. Interaction of herbicides with soil microbes in cassava. Indian Journal of Weed Science,29: 147150.

Rajkumar, R. 2013. A study on effect of salt stress in the seed germination and biochemical parameters of rice (Oryza sativa L.) under in vitro condition. Asian Journal Plant 
Sciences 3(6):20-25.

Raven PH, Evert RF, Eichhorn SE. 2005. Biology of Plants, $7^{\text {th }}$ Edition, W.H. Freeman and Company Publishers, New York, ISBN 0$7167-1007-2$.

Rennie, R.J. 1981. A Single medium for the isolation of acetylene reduction (nitrogen fixing) bacteria from soils. Canadian Journal of Microbiology, 27: 8-14.

Roger PA and Reynaud PA. 1979. Ecology of blue-green algae in paddy fields. Pages 289-309 in International Rice Research Institute. Nitrogen and rice. Los Bailos, Philippines

Sánchez-Baracaldo, Patricia; Raven, John A.; Pisani, Davide; Knoll, Andrew H. (201709-12). "Early photosynthetic eukaryotes inhabited low-salinity habitats". Proceedings of the National Academy of Sciences, 114 (37): E7737-E7745. doi: 10. 1073/pnas. 1620089114.

Shukla AK, Tiwari BK and Mishra RR. 1987. Effect of benomyl copper oxychloride and mancozeb on rhizosphere microflora of potato. Proceeding of the National Acadamy of Science, 53: 273 -278.

Sperber JE. 1958. Solubilization of apatite by soil microorganisms producing organic acids. Australian Journal of Agricultural Research, 9: 782 -787.

Standford S and English L. 1949. Use of flame photometer in rapid soil tests for $\mathrm{K}$ and Ca. Agronomy Journal, 41: 446 -447.

Subbiah, BV and Asija CJ. 1956. A rapid procedure for the estimation of available nitrogen in the soils. Current Science, 25: 359.

Upadhyay, RKand Panda SK. 2009. Copperinduced growth inhibition, oxidative stress and ultrastructural alterations in freshly grown water lettuce (Pistia stratiotes L.). C. R. Biologies., 332: 623-632.

Valsecchi, G, Gigliotti C and Farini A. 1995. Microbial biomass, activity and organic matter accumulation in soils contaminated with heavy metals. Biology and Fertility of Soils, 20: 253-259.

Vibhuti, Charu Shahi, Kiran Bargali and SS. Bargali. 2015. Seed germination and seedling growth parameters of rice (Oryza sativa $\mathrm{L}$.) varieties as affected by salt and water stress. Indian Journal of Agricultural Sciences 85(1): 102-108.

Wainwright M. 1978. A review of the effects of pesticides on microbial activity in soils. Journal of Soil Science, 29: 287 -298.

Windham AS and Windham MT. 2004. Chemical control of plant diseases. In: Plant Pathology. Concepts and Lab Exercises. CRC Pub.USA.

ZevenhuizenLPTM, Dolfing J, EshuisEJ and Scholten- Koerselman IJ. 1979. Inhibitory effects of copper on bacteria related to the free ion concentration. Microbial Ecology, 5: $139-146$.

\section{How to cite this article:}

Jeya Bharathi, M., K. Rajappan and Raju, M. 2021. Green Algae Diagnosis and Management in Low Land Paddy Fields of Cauvery Delta Zone, Tamil Nadu, India. Int.J.Curr.Microbiol.App.Sci. 10(02): 3407-3420. doi: https://doi.org/10.20546/ijcmas.2021.1002.376 